Annals of Pure and Applied Mathematics

Vol.19, No.2, 2019, 111-119

ISSN: 2279-087X (P), 2279-0888(online)

Published on 11 May 2019

www.researchmathsci.org

DOI: http://dx.doi.org/10.22457/apam.619v19n2a01

Annals of

Pure and Applied

Mathematics

\title{
All the Solutions of the Diophantine Equations $p^{x}+p^{y}=z^{2}$ and $p^{x}-p^{y}=z^{2}$ when $p \geq 2$ is Prime
} Nechemia Burshtein

117 Arlozorov Street, Tel - Aviv 6209814, Israel

Email: anb17@netvision.net.il

Received 6 May 2019; accepted 10 May 2019

Abstract. In this article, we present a comprehensive and fully detailed study of the equations $p^{x}+p^{y}=z^{2}$ and $p^{x}-p^{y}=z^{2}$ when $p \geq 2$ is prime, and $x, y, z$ are positive integers. For each equation we establish the cases of infinitely many solutions and of nosolutions. Several solutions are also exhibited.

Keywords: Diophantine equations, Catalan's Conjecture

AMS Mathematics Subject Classification (2010): 11D61

\section{Introduction}

The field of Diophantine equations is ancient, vast, and no general method exists to decide whether a given Diophantine equation has any solutions, or how many solutions.

The famous general equation

$$
p^{x}+q^{y}=z^{2}
$$

has many forms. The literature contains a very large number of articles on non-linear such individual equations involving particular primes and powers of all kinds. Among them are for example $[1,2,4,6]$.

In this paper, we consider the two equations

$$
\begin{aligned}
& p^{x}+p^{y}=z^{2} \\
& p^{x}-p^{y}=z^{2}
\end{aligned}
$$

in which $p \geq 2$ is prime, and $x, y, z$ are positive integers.

All other values introduced are also positive integers, unless otherwise specified.

In our discussion, we utilize Catalan's Conjecture.

In 1844, Catalan conjectured: The only solution in integers $r>0, s>0, a>1, b>1$ of the equation

is $r=b=3$ and $s=a=2$.

$$
r^{a}-s^{b}=1
$$

The conjecture was proven by Mihăilescu [5] in 2002. 


\section{Nechemia Burshtein}

In the first two sections, we consider $p^{x}+p^{y}=z^{2}$ when $p$ is odd and respectively when $p=2$, and in the latter two sections the equation $p^{x}-p^{y}=z^{2}$ when $p$ is odd, and respectively when $p=2$. Each section is self-contained, as are the cases in each section all of which are considered separately.

\section{All the solutions of $p^{x}+p^{y}=z^{2}$ when $p>2$ is prime}

In this section, we consider the equation $p^{x}+p^{y}=z^{2}$ in which $p$ is an odd prime. Except for $p=3$, it is established for any other prime that no solutions exist. When $p=3$, it is shown in Theorem 2.1 that the equation has infinitely many solutions demonstrated via an identity.

Theorem 2.1. Suppose $p$ is an odd prime, and $x, y, z$ are positive integers. Let $t=1$, $2, \ldots, k, \ldots$.

Then, only for $p=3$ there exist solutions of

given by

$$
p^{x}+p^{y}=z^{2}
$$

valid for each and every value $t \geq 1$.

$$
(p, x, y, z)=\left(3,2 t+1,2 t, 2 \cdot 3^{t}\right)
$$

The equation has infinitely many solutions.

Proof: From (1) we have that $z^{2}$ is even, and denote $z=2 T$. If $x=y$, then (1) yields $2 p^{\mathrm{x}}=z^{2}=4 T^{2}$ which is impossible. Therefore $x \neq y$ and $x, y$ are distinct. Without loss of generality, we shall assume that $x>y$. Let $n=1,2, \ldots, k, \ldots$. We shall express the values $x, y$ in terms of $t, n$. It follows that four cases exist, namely:
(a) $x>y, \quad x=2 t$,
$y=2 n+1$.
(b) $x>y, \quad x=2 t$,
$y=2 n$.
(c) $x>y, \quad x=2 t+1, \quad y=2 n$.
(d) $x>y, \quad x=2 t+1, \quad y=2 n+1$.

Suppose (a): $x>y, x=2 t, y=2 n+1$.

Denote $2 t=(2 n+1)+a$. If (1) has a solution, then

$$
p^{2 t}+p^{2 n+1}=p^{(2 n+1)+a}+p^{2 n+1}=p^{2 n+1}\left(p^{a}+1\right)=z^{2} .
$$

The value $p^{2 n+1}$ is not a square, and $p^{a}+1$ is not a multiple of $p$. It therefore follows that $p^{2 n+1}\left(p^{a}+1\right) \neq z^{2}$. Case (a) has no solutions.

Suppose (b): $x>y, x=2 t, y=2 n$.

Denote $2 t=(2 n)+b$ where $b$ is even, and let $b=2 h$. If (1) has a solution, then

$$
p^{2 t}+p^{2 n}=p^{2 n}\left(p^{b}+1\right)=\left(p^{n}\right)^{2}\left(p^{b}+1\right)=z^{2} \text {. }
$$

The factor $p^{b}+1$ must equal a square. Denote $p^{b}+1=Q^{2}$ or $Q^{2}-p^{b}=1$. Hence,

$$
Q^{2}-p^{b}=Q^{2}-p^{2 h}=Q^{2}-\left(p^{h}\right)^{2}=\left(Q-p^{h}\right)\left(Q+p^{h}\right)=1
$$

which is impossible. Thus, $p^{b}+1 \neq Q^{2}$ and $\left(p^{n}\right)^{2}\left(p^{b}+1\right) \neq z^{2}$. Case (b) has no solutions.

Suppose (c): $x>y, x=2 t+1, y=2 n$.

Denote $2 t+1=(2 n)+c$ where $c \geq 1$ is odd. If (1) has a solution, then

$$
p^{2 t+1}+p^{2 n}=p^{2 n}\left(p^{c}+1\right)=\left(p^{n}\right)^{2}\left(p^{c}+1\right)=z^{2} \text {. }
$$


All the Solutions of the Diophantine Equations $p^{x}+p^{y}=z^{2}$ and $p^{x}-p^{y}=z^{2}$ when $p \geq 2$ is Prime

The factor $p^{c}+1$ must be a square, say $p^{c}+1=G^{2}$. Then $p^{c}=G^{2}-1=(G-1)(G+1)$. Denote

$$
G-1=p^{A}, \quad G+1=p^{B}, \quad A<B, \quad A+B=c .
$$

Then $(G+1)-(G-1)=2=p^{A}\left(p^{B-A}-1\right)$, whose only solution when $p$ is odd is $A=0$, $p=3, B=1$. Hence $c=1$. It then follows that $n=t$, and $x, y$ are two consecutive integers. Thus, the identity

$$
3^{2 t+1}+3^{2 t}=3^{2 t}(3+1)=\left(3^{t}\right)^{2} \cdot 2^{2}=\left(2 \cdot 3^{t}\right)^{2}=z^{2}
$$

is valid for each and every value $t \geq 1$. The equation $p^{x}+p^{y}=z^{2}$ has infinitely many solutions of the form

$$
(p, x, y, z)=\left(3,2 t+1,2 t, 2 \cdot 3^{t}\right),
$$

and each value $t$ represents a unique solution.

This concludes case (c).

Suppose (d): $x>y, x=2 t+1, y=2 n+1$.

Denote $2 t+1=(2 n+1)+d$. If (1) has a solution, then

$$
p^{2 t+1}+p^{2 n+1}=p^{(2 n+1)+d}+p^{2 n+1}=p^{2 n+1}\left(p^{d}+1\right)=z^{2} \text {. }
$$

Since $p^{2 n+1}$ is not a square, and $p^{d}+1$ is not a multiple of $p$, it follows that $p^{2 n+1}\left(p^{d}+1\right) \neq z^{2}$. Case (d) has no solutions.

The proof of Theorem 2.1 is complete.

Remark 2.1. In [1], the equation $3^{x}+q^{y}=z^{2}$ was considered. For the particular case $q=3$ with consecutive integers $x, y$, it was shown: For all even $x$ and $y=x-1$, $3^{x}+3^{y}=z^{2}$ has no solutions, whereas for all odd $x$ and $y=x-1,3^{x}+3^{y}=z^{2}$ has infinitely many solutions.

In this article (Theorem $2.1-(\mathbf{c})$ ), it is shown that $p^{x}+p^{y}=z^{2}$ has a solution only when $p=3$. The solution $(p, x, y, z)=\left(3,2 t+1,2 t, 2 \cdot 3^{t}\right)$ is valid for all values $t \geq 1$, and $x, y$ are consecutive integers. The equation has infinitely many solutions.

\section{All the solutions of $p^{x}+p^{y}=z^{2}$ when $p=2$}

Elementary Number Theory is a branch of Number Theory which uses elementary methods to solve equations having integral solutions and also other problems. As an example, we have Euclid ( $3^{\text {rd }}$ century B.C.) with his first proof that the set of prime numbers is infinite. This proof is considered today as one of the simplest and most elementary proofs, but also as one of the classical most beautiful and elegant proofs ever. The simplicity which characterizes our discussion in Section 2 is also pursued in the same manner in the sections ahead.

In Theorem 3.1 we determine all the solutions of the equation $2^{x}+2^{y}=z^{2}$. We begin with a simple useful lemma.

Lemma 3.1. The equation $2^{M}+1=N^{2}$ has the unique solution $M=3$ and $N=3$. 


\section{Nechemia Burshtein}

Proof: If $2^{M}+1=N^{2}$ has a solution in positive integers, then $2^{M}=N^{2}-1=(N-1)(N$ $+1)$. Denote $N-1=2^{u}$ and $N+1=2^{v}$ where $u+v=M$. Then

$$
(N+1)-(N-1)=2^{v}-2^{u}=2 \text {. }
$$

The difference of two distinct powers of 2 is even, and 2 is the smallest possible such difference. It is easily seen that this occurs only when $v=2$ and $u=1$. Thus, $2^{u}=2^{1}=N-1$ or $N=3$, which satisfies $2^{v}=2^{2}=N+1$, and implies that $2^{M}+$ $1=3^{2}$ or $M=3$. The solution $(M, N)=(3,3)$ is unique as asserted.

Remark 3.1. Certainly for $N^{2}-2^{M}=1$, we could have used Catalan's Conjecture to obtain the unique solution $M=N=3$. Nevertheless, a very simple proof provided the same result.

Theorem 3.1. Suppose $p=2$, and $x, y, z$ are positive integers. Then the solutions of the equation

$$
2^{x}+2^{y}=z^{2}
$$

are as follows.

If $x, y$ are equal:

(a) No solutions when $x, y$ are even.

(b) Infinitely many solutions when $x, y$ are odd.

If $x, y$ are distinct:

(c) No solutions when $x$ is even and $y$ is odd.

(d) No solutions when $x, y$ are even.

(e) Infinitely many solutions when $x$ is odd and $y$ is even.

(f) No solutions when $x, y$ are odd.

Proof: Let $t=1,2, \ldots, k, \ldots$.

The case $x=y$.

The values $x, y$ are expressed in terms of $t$.

(a) Suppose $x, y$ are even, and denote $x=y=2 t$. If (2) has a solution, then

$$
2^{x}+2^{y}=2^{2 t}+2^{2 t}=2 \cdot 2^{2 t}=2\left(2^{t}\right)^{2}=z^{2} \text {. }
$$

The factor $\left(2^{t}\right)^{2}$ is a square, but 2 is not. Therefore $2\left(2^{t}\right)^{2} \neq z^{2}$. Case (a) has no solutions.

(b) Suppose $x, y$ are odd, and denote $x=y=2 t+1$. If (2) has a solution, then

$$
2^{x}+2^{y}=2^{2 t+1}+2^{2 t+1}=2 \cdot 2^{2 t+1}=2^{2 \cdot} \cdot 2^{2 t}=\left(2^{t+1}\right)^{2}=z^{2}
$$

The identity

$$
2^{2 t+1}+2^{2 t+1}=\left(2^{t+1}\right)^{2}
$$

is valid for each and every value $t \geq 1$. In this case, the equation $2^{x}+2^{y}=z^{2}$ has infinitely many solutions of the form

$$
(2, x, y, z)=\left(2,2 t+1,2 t+1,2^{t+1}\right),
$$

where each value $t$ determines a unique solution.

This concludes (b), and the case $x=y$.

The case $x \neq y$. 
All the Solutions of the Diophantine Equations $p^{x}+p^{y}=z^{2}$ and $p^{x}-p^{y}=z^{2}$ when $p \geq 2$ is Prime

Let $n=1,2, \ldots, k, \ldots$. Since $x, y$ are distinct, then without loss of generality, we shall assume that $x>y$. Hereafter, the values $x, y$ shall be expressed in terms of $t, n$.

(c) $x>y, \quad x=2 t, \quad y=2 n+1$.

Denote $2 t=(2 n+1)+a$. If (2) has a solution, then

$$
2^{2 t}+2^{2 n+1}=2^{(2 n+1)+a}+2^{2 n+1}=2^{2^{n+1}}\left(2^{a}+1\right)=z^{2} .
$$

The value $2^{2 n+1}$ is not a square. Since $2^{a}+1$ is odd, it follows in $(3)$ that $2^{2 n+1}\left(2^{a}+1\right) \neq$ $z^{2}$. Case (c) has no solutions.

(d) $x>y, \quad x=2 t, \quad y=2 n$.

Denote $2 t=(2 n)+b$ where $b$ is even. If (2) has a solution, then

$$
2^{2 t}+2^{2 n}=2^{2 n}\left(2^{b}+1\right)=\left(2^{n}\right)^{2}\left(2^{b}+1\right)=z^{2} \text {. }
$$

The factor $\left(2^{n}\right)^{2}$ is a square. By Lemma 3.1 , the factor $2^{b}+1$ is a square only when $b=3$ which is in contradiction that a priori $b$ is even. In (4), it then follows that $\left(2^{n}\right)^{2}\left(2^{b}+1\right) \neq z^{2}$. Case (d) has no solutions.

(e) $x>y, \quad x=2 t+1, \quad y=2 n$.

Denote $2 t+1=(2 n)+c$ where $c$ is odd. If (2) has a solution, then

$$
2^{2 t+1}+2^{2 n}=2^{2 n}\left(2^{c}+1\right)=\left(2^{n}\right)^{2}\left(2^{c}+1\right)=z^{2} \text {. }
$$

The factor $\left(2^{n}\right)^{2}$ is a square. By Lemma 3.1 , the factor $2^{c}+1$ is a square only when $c=3$. Therefore in (5) with $c=3$ we obtain

The identity

$$
z^{2}=\left(2^{n}\right)^{2} \cdot 3^{2}=\left(3 \cdot 2^{n}\right)^{2} .
$$

$$
2^{2 n+3}+2^{2 n}=\left(3 \cdot 2^{n}\right)^{2}
$$

is valid for each and every value $n \geq 1$. Therefore in this case, the equation $2^{x}+2^{y}=z^{2}$ has infinitely many solutions of the form

$$
(2, x, y, z)=\left(2,2 n+3,2 n, 3 \cdot 2^{n}\right),
$$

where each value $n$ represents a unique solution.

This completes case (e).

(f) $x>y, \quad x=2 t+1, \quad y=2 n+1$.

Denote $2 t+1=(2 n+1)+d$. If (2) has a solution, then

$$
2^{2 t+1}+2^{2 n+1}=2^{(2 n+1)+d}+2^{2 n+1}=2^{2 n+1}\left(2^{d}+1\right)=z^{2} .
$$

The value $2^{2 n+1}$ is not a square. Since $2^{d}+1$ is odd, it follows in (6) that $2^{2 n+1}\left(2^{d}+1\right)$ $\neq z^{2}$. Case (f) has no solutions.

The proof of Theorem 3.1 is complete.

Remark 3.2. An interesting consequence follows from the established solutions of the equation $p^{x}+p^{y}=z^{2}$. When $p=3$ and $x>y$, then $z=2 \cdot 3^{t}$ where $t \geq 1$. Whereas when $p=2$ and $x>y$, then $z=3 \cdot 2^{n}$ where $n \geq 1$. Therefore, each of these solutions is exactly a multiple of $6^{2}$. 


\section{Nechemia Burshtein}

\section{All the solutions of $p^{x}-p^{y}=z^{2}$ when $p>2$ is prime}

For $p^{x}-p^{y}=z^{2}$ in which $p$ is an odd prime, we determine first the form of $p$, and then all the solutions of the equation. Some solutions are also exhibited.

Theorem 4.1. Suppose $p$ is an odd prime, and $x, y, z$ are positive integers. Let $t=$ $1,2, \ldots, k, \ldots$, and $v=1,2, \ldots, k, \ldots$. Let $v \geq 1$ be a fixed value for which $p=4 v^{2}+$ 1. Then for each such prime $p$, the equation

has a solution of the form

$$
p^{x}-p^{y}=z^{2}
$$

valid for all values $t \geq 1$.

$$
(p, x, y, z)=\left(4 v^{2}+1,2 t+1,2 t, 2 v \cdot\left(4 v^{2}+1\right)^{t}\right)
$$

The equation has infinitely many solutions.

Proof: Let $n=1,2, \ldots, k, \ldots$. The values $x, y$ are expressed in terms of $t, n$. Four cases then exist, namely:
(a) $x=2 t, \quad y=2 n+1$.
(b) $x=2 t, \quad y=2 n$.
(c) $x=2 t+1, \quad y=2 n$.
(d) $x=2 t+1, \quad y=2 n+1$.

In each of $(\mathbf{a})-(\mathbf{d})$, a priori $x>y$.

Suppose (a): $x=2 t, y=2 n+1$.

Denote $2 t=(2 n+1)+a$. If (7) has a solution, we obtain

$$
p^{2 t}-p^{2 n+1}=p^{(2 n+1)+a}-p^{2 n+1}=p^{2 n+1}\left(p^{a}-1\right)=z^{2} \text {. }
$$

The value $p^{2 n+1}$ is not a square. Since $p^{a}-1$ is not a multiple of $p$, it therefore follows that $p^{2 n+1}\left(p^{a}-1\right) \neq z^{2}$. Case (a) has no solutions.

Suppose (b): $x=2 t, y=2 n$.

Denote $2 t=(2 n)+b$ where $b$ is even, and let $b=2 s$. If (7) has a solution, we have

$$
p^{2 t}-p^{2 n}=p^{2 n}\left(p^{b}-1\right)=\left(p^{n}\right)^{2}\left(p^{b}-1\right)=z^{2} .
$$

Since $\left(p^{n}\right)^{2}$ is a square, $p^{b}-1$ must also equal a square, say $p^{b}-1=Q^{2}$, or $p^{b}-Q^{2}=1$. Hence,

$$
p^{b}-Q^{2}=p^{2 s}-Q^{2}=\left(p^{s}\right)^{2}-Q^{2}=\left(p^{s}-Q\right)\left(p^{s}+Q\right)=1
$$

which is impossible. Hence $p^{b}-1 \neq Q^{2}$ and $\left(p^{n}\right)^{2}\left(p^{b}-1\right) \neq z^{2}$. Case (b) has no solutions.

Suppose (c): $x=2 t+1, y=2 n$.

Denote $2 t+1=(2 n)+c$ where $c$ is odd. If (7) has a solution, then

$$
p^{2 t+1}-p^{2 n}=p^{2 n}\left(p^{c}-1\right)=\left(p^{n}\right)^{2}\left(p^{c}-1\right)=z^{2} \text {. }
$$

The factor $p^{c}-1$ must equal a square, say $p^{c}-1=G^{2}$. For all odd $c>1$, the equation $p^{c}-1=G^{2}$ or $p^{c}-G^{2}=1$, in which $p>0, G>0, c>1,2>1$ satisfy the conditions of Catalan's Conjecture. However, the unique solution $(3,2,2,3)$ of Catalan's Conjecture is not a solution of $p^{c}-G^{2}=1$. Therefore, $p^{c}-1 \neq G^{2}$ when $c>1$ is odd. 
All the Solutions of the Diophantine Equations $p^{x}+p^{y}=z^{2}$ and $p^{x}-p^{y}=z^{2}$ when $p \geq 2$ is Prime

When $c=1$, it follows that $t=n$ and $p^{1}-1=G^{2}$ where $G$ is even. Each prime $p$ is of the form either $4 N+3$ or $4 N+1$. If $p=4 N+3$, then $p-1=4 N+2$ $=2(2 N+1) \neq G^{2}$. Therefore, $p=4 N+1$ and $N=v^{2}$ yield $p=4 v^{2}+1$, where $G^{2}=4 v^{2}=(2 v)^{2}$. Moreover, $x, y$ are two consecutive integers. The value $z$ is determined accordingly, and $z=2 v \cdot\left(4 v^{2}+1\right)^{t}$.

Finally, for any fixed prime $p=4 v^{2}+1(v \geq 1)$, with all values $t \geq 1$ in $x=$ $2 t+1, \quad y=2 t$, one obtains infinitely many solutions of (7). Furthermore, the equation $p^{x}-p^{y}=z^{2}$ then has infinitely many solutions for each and every prime $p=4 v^{2}+1$ as follows

$$
(p, x, y, z)=\left(4 v^{2}+1,2 t+1,2 t, 2 v \cdot\left(4 v^{2}+1\right)^{t}\right) \text {. }
$$

There are infinitely many primes $p=4 N+1$, as there are also infinitely many primes of the form $p=4 v^{2}+1$. Case (c) is complete.

For the convenience of the reader we exhibit some solutions of case (c). The first four primes of the form $p=4 v^{2}+1$ are $p=5,17,37,101$, and some solutions are:

Solution 1. $\quad 5^{3}-5^{2}=10^{2}$.

Solution 2. $\quad 5^{5}-5^{4}=50^{2}$.

Solution 3. $\quad 5^{7}-5^{6}=250^{2}$.

Solution 4. $\quad 17^{3}-17^{2}=68^{2}$.

Solution 5. $17^{5}-17^{4}=1156^{2}$.

Solution 6. $\quad 37^{3}-37^{2}=222^{2}$.

Solution 7. $101^{3}-101^{2}=1010^{2}$.

Suppose (d): $x=2 t+1, \quad y=2 n+1$.

Denote $2 t+1=(2 n+1)+d$. If $(7)$ has a solution, then

$$
p^{2 t+1}-p^{2 n+1}=p^{(2 n+1)+d}-p^{2 n+1}=p^{2 n+1}\left(p^{d}-1\right)=z^{2} \text {. }
$$

The value $p^{2 n+1}$ is not a square. Since $p^{d}-1$ is not a multiple of $p$, it therefore follows that $p^{2 n+1}\left(p^{d}-1\right) \neq z^{2}$. Case (d) has no solutions.

This concludes the proof of Theorem 4.1.

\section{All the solutions of $p^{x}-p^{y}=z^{2}$ when $p=2$}

In this section, we determine all the solutions of the equation $p^{x}-p^{y}=z^{2}$ when $p=2$. This is done in the following theorem. 


\section{Nechemia Burshtein}

Theorem 5.1. Suppose $p=2$, and $x, y, z$ are positive integers. Then the solutions of the equation

$$
2^{x}-2^{y}=z^{2}
$$

are as follows.

(a) No solutions when $x$ is even and $y$ is odd.

(b) No solutions when $x, y$ are even.

(c) Infinitely many solutions when $x$ is odd and $y$ is even.

(d) No solutions when $x, y$ are odd.

Proof: In each of $(\mathbf{a})-(\mathbf{d})$, a priori $x>y$. Let $t=1,2, \ldots, k, \ldots$, and $n=1,2, \ldots$, $k, \ldots$ We shall express the values $x, y$ in terms of $t, n$.

Suppose (a): $x=2 t, y=2 n+1$.

Denote $2 t=(2 n+1)+a$. If (8) has a solution, then

$$
2^{2 t}-2^{2 n+1}=2^{(2 n+1)+a}-2^{2 n+1}=2^{2 n+1}\left(2^{a}-1\right)=z^{2} .
$$

The value $2^{2 n+1}$ is not equal to a square. The factor $2^{a}-1$ is odd. Hence, $2^{2 n+1}\left(2^{a}-1\right) \neq$ $z^{2}$. Case (a) has no solutions.

Suppose (b): $x=2 t, \quad y=2 n$.

Denote $2 t=(2 n)+b$ where $b$ is even, and let $b=2 r$. If (8) has a solution, then

$$
2^{2 t}-2^{2 n}=2^{2 n}\left(2^{b}-1\right)=\left(2^{n}\right)^{2}\left(2^{b}-1\right)=z^{2} .
$$

Since $\left(2^{n}\right)^{2}$ is a square, $2^{b}-1$ must also equal a square. Let $2^{b}-1=L^{2}$ or $2^{b}-L^{2}=1$. Hence,

$$
2^{b}-L^{2}=2^{2 r}-L^{2}=\left(2^{r}\right)^{2}-L^{2}=\left(2^{r}-L\right)\left(2^{r}+L\right)=1
$$

which is impossible. Therefore, $2^{b}-1 \neq L^{2}$ and $\left(2^{n}\right)^{2}\left(2^{b}-1\right) \neq z^{2}$. Case (b) has no solutions.

Suppose (c): $x=2 t+1, \quad y=2 n$.

Denote $2 t+1=(2 n)+c$ where $c$ is odd. If (8) has a solution, then

$$
2^{2 t+1}-2^{2 n}=2^{2 n}\left(2^{c}-1\right)=\left(2^{n}\right)^{2}\left(2^{c}-1\right)=z^{2}
$$

implying that $2^{c}-1$ equals a square, say $2^{c}-1=K^{2}$ and $K$ is odd. Suppose that $c>1$ is odd. The equation $2^{c}-1=K^{2}$ yields $2^{c}-K^{2}=1$, and the values $2>0, K>0, c>1$, $2>1$ satisfy the conditions of Catalan's Conjecture. But, the unique solution $(3,2,2,3)$ of Catalan's Conjecture is not a solution of $2^{c}-K^{2}=1$. Therefore, $2^{c}-1 \neq K^{2}$ when $c>1$ is odd.

Hence, if (9) has any solutions, then $c=1$. When $c=1$, then in (9) $2^{c}-1=1$ $(K=1)$, and $z=2^{n}$. Moreover, $t=n$, and $x, y$ are consecutive integers.

The equation $2^{x}-2^{y}=z^{2}$ has infinitely many solutions

$$
(2, x, y, z)=\left(2,2 n+1,2 n, 2^{n}\right)
$$

valid for each and every value $n \geq 1$. Case (c) is complete.

Suppose (d): $x=2 t+1, \quad y=2 n+1$.

Denote $2 t+1=(2 n+1)+d$. If $(8)$ has a solution, then

$$
2^{2 t+1}-2^{2 n+1}=2^{(2 n+1)+d}-2^{2 n+1}=2^{2 n+1}\left(2^{d}-1\right)=z^{2} \text {. }
$$


All the Solutions of the Diophantine Equations $p^{x}+p^{y}=z^{2}$ and $p^{x}-p^{y}=z^{2}$ when $p \geq 2$ is Prime

The value $2^{2 n+1}$ is not a square, and $2^{d}-1$ is odd. It then follows that $2^{2 n+1}\left(2^{d}-1\right)$ $\neq z^{2}$. Case (d) has no solutions.

This completes the proof of Theorem 5.1.

Final Remark. An interesting fact is revealed from the solutions established in Theorems $2.1-5.1$. The value $x$ is odd in all the solutions, whereas the value $y$ is odd in Theorem $3.1-(\mathbf{b})$, but even in all other solutions.

\section{REFERENCES}

1. N. Burshtein, On solutions to the diophantine equation $3^{x}+q^{y}=z^{2}$, Annals of Pure and Applied Mathematics, 19 (2) (2019) 169 - 173.

2. N. Burshtein, On solutions of the diophantine equations $p^{3}+q^{3}=z^{2}$ and $p^{3}-q^{3}=z^{2}$ when $p, q$ are primes, Annals of Pure and Applied Mathematics, 18 (1) (2018) $51-57$.

3. N. Burshtein, On the infinitude of solutions to the diophantine equation $p^{x}+q^{y}=z^{2}$ when $p=2$ and $p=3$, Annals of Pure and Applied Mathematics, 13 (2) (2017) $207-210$.

4. S. Chotchaisthit, On the diophantine equation $p^{x}+(p+1)^{y}=z^{2}$ when $p$ is a Mersenne Prime, Int. J. Pure Appl. Math., 88 (2) (2013) 169 - 172.

5. P. Mihăilescu, Primary cyclotomic units and a proof of Catalan's conjecture, $J$. Reine Angew. Math., 572 (2004) 167- 195.

6. B. Poonen, Some diophantine equations of the form $x^{n}+y^{n}=z^{m}$, Acta Arith., 86 (1998) $193-205$.

7. B. Sroysang, On the diophantine equation $5^{x}+7^{y}=z^{2}$, Int. J. Pure Appl. Math., 89 (2013) $115-118$. 